\title{
Marichev-Saigo-Maeda Fractional Integration Operators Involving Generalized Bessel Functions
}

\author{
Saiful R. Mondal ${ }^{1}$ and K. S. Nisar ${ }^{2}$ \\ ${ }^{1}$ Department of Mathematics \& Statistics, College of Science, King Faisal University, P.O. Box 400, Hofuf, Al-Ahsa 31982, Saudi Arabia \\ ${ }^{2}$ Department of Mathematics, College of Arts and Science, Salman bin Abdulaziz University, P.O. Box 54, Wadi Al-Dawaser 11991, \\ Saudi Arabia
}

Correspondence should be addressed to Saiful R. Mondal; saiful786@gmail.com

Received 12 February 2014; Accepted 4 March 2014; Published 8 April 2014

Academic Editor: Santanu Saha Ray

Copyright (c) 2014 S. R. Mondal and K. S. Nisar. This is an open access article distributed under the Creative Commons Attribution License, which permits unrestricted use, distribution, and reproduction in any medium, provided the original work is properly cited.

Two integral operators involving Appell's functions, or Horn's function in the kernel are considered. Composition of such functions with generalized Bessel functions of the first kind is expressed in terms of generalized Wright function and generalized hypergeometric series. Many special cases, including cosine and sine function, are also discussed.

\section{Introduction}

Let $\alpha, \alpha^{\prime}, \beta, \beta^{\prime}, \gamma \in \mathbb{C}$ and $x>0$; then the generalized fractional integral operators involving Appell's functions or Horn's function are defined as follows:

$$
\begin{aligned}
& \left(I_{0,+}^{\alpha, \alpha^{\prime}, \beta, \beta^{\prime}, \gamma} f\right)(x) \\
& =\frac{x^{-\alpha}}{\Gamma(\gamma)} \int_{0}^{x}(x-t)^{\gamma-1} t^{-\alpha^{\prime}} F_{3} \\
& \quad \times\left(\alpha, \alpha^{\prime}, \beta, \beta^{\prime} ; \gamma ; 1-\frac{t}{x}, 1-\frac{x}{t}\right) f(t) d t, \\
& \left(I_{0,-}^{\alpha, \alpha^{\prime}, \beta, \beta^{\prime}, \gamma} f\right)(x) \\
& =\frac{x^{-\alpha^{\prime}}}{\Gamma(\gamma)} \int_{x}^{\infty}(t-x)^{\gamma-1} t^{-\alpha} F_{3} \\
& \quad \times\left(\alpha, \alpha^{\prime}, \beta, \beta^{\prime} ; \gamma ; 1-\frac{t}{x}, 1-\frac{x}{t}\right) f(t) d t,
\end{aligned}
$$

with $\operatorname{Re}(\gamma)>0$. The generalized fractional integral operators of the types (1) and (2) have been introduced by Marichev [1] and later extended and studied by Saigo and Maeda [2]. These operators together are known as the Marichev-Saigo-Maeda operator.
The fractional integral operator has many interesting applications in various subfields in applicable mathematical analysis; for example, [3], it has applications related to a certain class of complex analytic functions. The results given in [4-6] can be referred to for some basic results on fractional calculus.

The purpose of this work is to investigate compositions of integral transforms (1) and (2) with the generalized Bessel function of the first kind $\mathscr{W}_{p, b, c}$ defined for complex $z \in \mathbb{C}$ and $b, c, p \in \mathbb{C}$ by

$$
\mathscr{W}_{p, b, c}(z)=\sum_{k=0}^{\infty} \frac{(-1)^{k} c^{k}}{\Gamma(\kappa+k) k !}\left(\frac{z}{2}\right)^{2 k+p}
$$

where $\kappa:=p+(b+1) / 2$. More details related to the function $\mathscr{W}_{p, b, c}$ and its particular cases can be found in $[7,8]$ and references therein. It is worth mentioning that $\mathscr{W}_{p, 1,1}=J_{p}$ is Bessel function of order $p$ and $\mathscr{W}_{p, 1,-1}=I_{p}$ is modified Bessel function of order $p$. Also, $\mathscr{W}_{p, 2,1}=2 j_{p} / \sqrt{\pi}$ is spherical Bessel function of order $p$ and $\mathscr{W}_{p, 2,-1}=2 i_{p} / \sqrt{\pi}$ is modified spherical Bessel function of order $p$. Thus the study of the integral transform of $\mathscr{W}_{p, b, c}$ will give far reaching results than the result in $[9,10]$.

The present paper is organized as follows. In Sections 2 and 3, composition of integral transforms (1) and (2) with 
generalized Bessel function (3) is given in terms of generalized Wright functions and generalized hypergeometric functions, respectively. Special cases like $p=-b / 2(p=1-$ $b / 2$ ) of $\mathscr{W}_{p, b, c}$ give the composition of (1) and (2) with cosine and hyperbolic cosine (sine and hyperbolic sine) functions, which are discussed in Section 4. Some concluding remarks and comparison with earlier known work are mentioned in Section 5.

The following two results given by Saigo et al. $[2,11]$ are needed in sequel.

Lemma 1. Let $\alpha, \alpha^{\prime}, \beta, \beta^{\prime}, \gamma, \rho \in \mathbb{C}$ be such that $\operatorname{Re}(\gamma)>0$ and

$$
\operatorname{Re}(\rho)>\max \left\{0, \operatorname{Re}\left(\alpha-\alpha^{\prime}-\beta-\gamma\right), \operatorname{Re}\left(\alpha^{\prime}-\beta^{\prime}\right)\right\}
$$

Then there exists the relation

$$
\begin{aligned}
& \left(I_{0,+}^{\alpha, \alpha^{\prime}, \beta, \beta^{\prime}, \gamma} t^{\rho-1}\right)(x) \\
& \quad=\Gamma\left[\begin{array}{c}
\rho, \rho+\gamma-\alpha-\alpha^{\prime}-\beta, \rho+\beta^{\prime}-\alpha^{\prime} \\
\rho+\beta^{\prime}, \rho+\gamma-\alpha-\alpha^{\prime}, \rho+\gamma-\alpha^{\prime}-\beta
\end{array}\right] x^{\rho-\alpha-\alpha^{\prime}+\gamma-1},
\end{aligned}
$$

where

$$
\Gamma\left[\begin{array}{l}
a, b, c \\
d, e, f
\end{array}\right]=\frac{\Gamma(a) \Gamma(b) \Gamma(c)}{\Gamma(d) \Gamma(e) \Gamma(f)}
$$

Lemma 2. Let $\alpha, \alpha^{\prime}, \beta, \beta^{\prime}, \gamma, \rho \in \mathbb{C}$ be such that $\operatorname{Re}(\gamma)>0$ and

$$
\begin{aligned}
& \operatorname{Re}(\rho) \\
& \quad<1+\min \left\{\operatorname{Re}(-\beta), \operatorname{Re}\left(\alpha+\alpha^{\prime}-\gamma\right), \operatorname{Re}\left(\alpha+\beta^{\prime}-\gamma\right)\right\} .
\end{aligned}
$$

Then there exists the relation

$$
\begin{aligned}
& \left(I_{0,+}^{\alpha, \alpha^{\prime}, \beta, \beta^{\prime}, \gamma} t^{\rho-1}\right)(x) \\
& =\Gamma\left[\begin{array}{c}
1-\rho-\gamma+\alpha+\alpha^{\prime}, 1-\rho+\alpha+\beta^{\prime}, 1-\rho-\beta \\
1-\rho, 1-\rho+\alpha+\alpha^{\prime}+\beta+\beta^{\prime}-\gamma, 1-\rho+\alpha-\beta
\end{array}\right] \\
& \quad \times x^{\rho-\alpha-\alpha^{\prime}+\gamma-1} .
\end{aligned}
$$

\section{Representations in terms of Generalized Wright Functions}

In this section composition of integral transforms (1) and (2) with generalized Bessel function (3) is given in terms of the generalized Wright hypergeometric function ${ }_{p} \psi_{q}(z)$ which is defined by the series

$$
\left.{ }_{p} \psi_{q}(z)={ }_{p} \psi_{q}\left[\begin{array}{c}
\left(a_{i}, \alpha_{i}\right)_{1, p} \\
\left(b_{j}, \beta_{j}\right)_{1, q}
\end{array}\right] z\right]=\sum_{k=0}^{\infty} \frac{\prod_{i=1}^{p} \Gamma\left(a_{i}+\alpha_{i} k\right)}{\prod_{j=1}^{q} \Gamma\left(b_{j}+\beta_{j} k\right)} \frac{z^{k}}{k !} .
$$

Here $a_{i}, b_{j} \in \mathbb{C}$, and $\alpha_{i}, \beta_{j} \in \mathbb{R}(i=1,2, \ldots, p ; j=$ $1,2, \ldots, q)$. Asymptotic behavior of this function for large values of argument of $z \in \mathbb{C}$ was studied in [12] and under the condition

$$
\sum_{j=1}^{q} \beta_{j}-\sum_{i=1}^{p} \alpha_{i}>-1
$$

in $[13,14]$. Properties of this generalized Wright function were investigated in $[9,15,16]$. In particular, it was proved [15] that ${ }_{p} \psi_{q}(z), z \in \mathbb{C}$, is an entire function under the condition (10). Interesting results related to generalized Wright functions are also given in [17].

Theorem 3. Let $\alpha, \alpha^{\prime}, \beta, \beta^{\prime}, \gamma, \rho, p, b, c \in \mathbb{C}$ such that $\kappa \neq 0,-1,-2, \ldots$. Suppose that $\operatorname{Re}(\gamma)>0$ and $\operatorname{Re}(\rho+p)>$ $\max \left\{0, \operatorname{Re}\left(\alpha+\alpha^{\prime}+\beta-\gamma\right), \operatorname{Re}\left(\alpha^{\prime}-\beta^{\prime}\right)\right\}$. Then

$$
\begin{aligned}
& \left(I_{0,+}^{\alpha, \alpha^{\prime}, \beta, \beta^{\prime}, \gamma} t^{\rho-1} \mathscr{W}_{p, b, c}(t)\right)(x) \\
& \quad=\frac{x^{\rho+p-\alpha-\alpha^{\prime}+\gamma-1}}{2^{p}} \times{ }_{3} \psi_{4}\left[\begin{array}{cc}
(\rho+p, 2),\left(\rho+p+\gamma-\alpha-\alpha^{\prime}-\beta, 2\right)\left(\rho+p+\beta^{\prime}-\alpha^{\prime}, 2\right) ; & \\
\left(\rho+p+\beta^{\prime}, 2\right),\left(\rho+p+\gamma-\alpha-\alpha^{\prime}, 2\right), & \mid-\frac{c x^{2}}{4} \\
\left(\rho+p+\gamma-\alpha^{\prime}-\beta, 2\right),(\kappa, 1) &
\end{array}\right] .
\end{aligned}
$$

Proof. An application of integral transform (1) to the generalized Bessel function (3) leads to the formula

$$
\begin{aligned}
& \left(I_{0,+}^{\alpha, \alpha^{\prime}, \beta, \beta^{\prime}, \gamma} t^{\rho-1} \mathscr{W}_{p, b, c}(t)\right)(x) \\
& \quad=\left(I_{0,+}^{\alpha, \alpha^{\prime}, \beta, \beta^{\prime}, \gamma} \sum_{k=0}^{\infty} \frac{(-c)^{k}(1 / 2)^{2 k+p}}{\Gamma(\kappa+k) k !} t^{\rho+p+2 k-1}\right)(x) .
\end{aligned}
$$

Now changing the order of integration and summation in right-hand side of (12) yields

$$
\begin{aligned}
& \left(I_{0,+}^{\alpha, \alpha^{\prime}, \beta, \beta^{\prime}, \gamma} t^{\rho-1} \mathscr{W}_{p, b, c}(t)\right)(x) \\
& \quad=\sum_{k=0}^{\infty} \frac{(-c)^{k}(1 / 2)^{2 k+p}}{\Gamma(\kappa+k) k !}\left(I_{0,+}^{\alpha, \alpha^{\prime}, \beta, \beta^{\prime}, \gamma} t^{\rho+p+2 k-1}\right)(x) .
\end{aligned}
$$


Note that, for all $k=0,1,2, \ldots$,

$$
>\max \left\{0, \operatorname{Re}\left(\alpha-\alpha^{\prime}-\beta-\gamma\right), \operatorname{Re}\left(\alpha^{\prime}-\beta^{\prime}\right)\right\} .
$$

$$
\begin{array}{r}
\operatorname{Re}(\rho+p+2 k) \\
\geq \operatorname{Re}(\rho+p)
\end{array}
$$

$$
\begin{aligned}
& \left(I_{0,+}^{\alpha, \alpha^{\prime} \beta, \beta^{\prime}, \gamma} t^{\rho-1} \mathscr{W}_{p, b, c}(t)\right)(x) \\
& \quad=\frac{x^{\rho+p-\alpha-\alpha^{\prime}+\gamma-1}}{2^{p}} \times \sum_{k=0}^{\infty} \Gamma\left[\begin{array}{c}
\rho+p+2 k, \rho+p+\gamma-\alpha-\alpha^{\prime}-\beta+2 k, \rho+p+\beta^{\prime}-\alpha^{\prime}+2 k \\
\rho+p+\beta^{\prime}+2 k, \rho+p+\gamma-\alpha-\alpha^{\prime}+2 k, \rho+p+\gamma-\alpha^{\prime}-\beta+2 k, \kappa+k
\end{array}\right] \frac{1}{k !}\left(\begin{array}{c}
c x^{2} \\
4
\end{array}\right)^{k} .
\end{aligned}
$$

Interpreting the right-hand side of (15), the equality (11) can be obtained from (6) and then by using the definition of generalized Wright function.
Theorem 4. Let $\alpha, \alpha^{\prime}, \beta, \beta^{\prime}, \gamma, \rho, p, b, c \in \mathbb{C}$ such that $\kappa \neq 0,-1,-2, \ldots$. Suppose that $\operatorname{Re}(\gamma)>0$ and $\operatorname{Re}(\rho-p)<$ $1+\min \left\{\operatorname{Re}(-\beta), \operatorname{Re}\left(\alpha+\alpha^{\prime}-\gamma\right), \operatorname{Re}\left(\alpha+\beta^{\prime}-\gamma\right)\right\}$. Then

$$
\begin{aligned}
& \left(I_{0,-}^{\alpha, \alpha^{\prime}, \beta, \beta^{\prime}, \gamma} t^{\rho-1} \mathscr{W}_{p, b, c}\left(\frac{1}{t}\right)\right)(x) \\
& \quad=\frac{x^{\rho-p-\alpha-\alpha^{\prime}+\gamma-1}}{2^{p}} \times{ }_{3} \psi_{4}\left[\begin{array}{c}
\left(1-\rho+p-\gamma+\alpha+\alpha^{\prime}, 2\right),\left(1-\rho+p+\alpha-\beta^{\prime}-\gamma, 2\right)(1-\rho+p-\beta, 2) \\
(1-\rho+p, 2),\left(1-\rho+p-\gamma+\alpha+\alpha^{\prime}+\beta^{\prime}, 2\right),(1-\rho+p+\alpha-\beta, 2),(\kappa, k)
\end{array}\right.
\end{aligned}
$$

Proof. Using (2) and (3) and then changing the order of integration and summation, which is justified under the conditions with Theorem 4, yield

$$
\begin{aligned}
& \left(I_{0,-}^{\alpha, \alpha^{\prime}, \beta, \beta^{\prime}, \gamma} t^{\rho-1} \mathscr{W}_{p, b, c}\left(\frac{1}{t}\right)\right)(x) \\
& \quad=\sum_{k=0}^{\infty} \frac{(-c)^{k}(1 / 2)^{2 k+p}}{\Gamma(\kappa+k) k !}\left(I_{0,-}^{\alpha, \alpha^{\prime}, \beta, \beta^{\prime}, \gamma} t^{\rho-p-2 k-1}\right)(x) .
\end{aligned}
$$

Note that, for all $k=0,1,2, \ldots$,

$$
\begin{aligned}
& \operatorname{Re}(\rho-p-2 k) \\
& \quad \leq \operatorname{Re}(\rho-p) \\
& \quad<1+\min \left\{\operatorname{Re}(-\beta), \operatorname{Re}\left(\alpha+\alpha^{\prime}-\gamma\right), \operatorname{Re}\left(\alpha+\beta^{\prime}-\gamma\right)\right\} .
\end{aligned}
$$

Hence replacing $\rho$ by $\rho-p-2 k$ in Lemma 2 and using (8), we obtain

$$
\begin{aligned}
& \left(I_{0,-}^{\alpha, \alpha^{\prime}, \beta, \beta^{\prime}, \gamma} t^{\rho-1} \mathscr{W}_{p, b, c}\left(\frac{1}{t}\right)\right)(x) \\
& \quad=\frac{x^{\rho-p-\alpha-\alpha^{\prime}+\gamma-1}}{2^{p}} \times \sum_{k=0}^{\infty} \Gamma\left[\begin{array}{c}
1-\rho+p-\gamma+\alpha+\alpha^{\prime}+2 k, 1-\rho+p+\alpha+\beta^{\prime}-\gamma+2 k, 1-\rho+p-\beta+2 k \\
1-\rho+p+2 k, 1-\rho+p-\gamma+\alpha+\alpha^{\prime}+\beta^{\prime}+2 k, 1-\rho+p+\alpha-\beta+2 k
\end{array}\right] \frac{1}{k !}\left(-\frac{c}{4 x^{2}}\right)^{k} .
\end{aligned}
$$


Now (6), (9), and (19) together imply that

$$
\begin{aligned}
& \left(I_{0,-}^{\alpha, \alpha^{\prime}, \beta, \beta^{\prime}, \gamma} t^{\rho-1} \mathscr{W}_{p, b, c}\left(\frac{1}{t}\right)\right)(x) \\
& \quad=\frac{x^{\rho-p-\alpha-\alpha^{\prime}+\gamma-1}}{2^{p}} \times{ }_{3} \psi_{4}\left[\begin{array}{c}
\left(1-\rho+p-\gamma+\alpha+\alpha^{\prime}, 2\right),\left(1-\rho+p+\alpha-\beta^{\prime}-\gamma, 2\right)(1-\rho+p-\beta, 2) ; \\
(1-\rho+p, 2),\left(1-\rho+p-\gamma+\alpha+\alpha^{\prime}+\beta^{\prime}, 2\right),(1-\rho+p+\alpha-\beta, 2),(p, k)
\end{array} \quad \begin{array}{c}
\mid-\frac{c}{4 x^{2}}
\end{array}\right],
\end{aligned}
$$

and this completes the proof.

\section{Representation in terms of Generalized Hypergeometric Series}

The generalized hypergeometric function ${ }_{p} F_{q}\left(a_{1}, \ldots, a_{p} ; c_{1}\right.$, $\left.\ldots, c_{q} ; z\right)$ is given by the representation

$$
\begin{aligned}
{ }_{p} F_{q} & \left(a_{1}, \ldots, a_{p} ; c_{1}, \ldots, c_{q} ; z\right) \\
& =\sum_{k=0}^{\infty} \frac{\left(a_{1}\right)_{k} \cdots\left(a_{p}\right)_{k}}{\left(c_{1}\right)_{k} \cdots\left(c_{q}\right)_{k}} \frac{z^{k}}{k !}, \quad(z \in \mathbb{C}),
\end{aligned}
$$

where none of the denominator parameters is zero or a negative integer. Here $p$ or $q$ are allowed to be zero. The series (21) is convergent for all finite $z$ if $p \leq q$, while, for $p=q+1$, it is convergent for $|z|<1$ and divergent for $|z|>1$.
Results obtained in this section demonstrate the image formula for the generalized Bessel functions $\mathscr{W}_{p, b, c}$ under the operators (1) and (2) in terms of generalized hypergeometric functions. The well-known Legendre duplication formulas [18] given by

$$
\begin{gathered}
\Gamma(2 z)=\frac{2^{2 z-1}}{\sqrt{\pi}} \Gamma(z) \Gamma\left(z+\frac{1}{2}\right), \\
(z)_{2 k}=2^{2 k}\left(\frac{z}{2}\right)_{k}\left(\frac{z+1}{2}\right)_{k}, \quad\left(k \in \mathbb{N}_{0}\right),
\end{gathered}
$$

are required for this purpose.

Theorem 5. Let $\alpha, \alpha^{\prime}, \beta, \beta^{\prime}, \gamma, \rho, p, b, c \in \mathbb{C}$ such that $\kappa \neq-$ $1,-2, \ldots$. Suppose that $\operatorname{Re}(\gamma)>0$ and $\operatorname{Re}(\rho+p)>$ $\max \left\{0, \operatorname{Re}\left(\alpha-\alpha^{\prime}-\beta-\gamma\right), \operatorname{Re}\left(\alpha^{\prime}-\beta^{\prime}\right)\right\}$. Then the following formula holds:

$$
\begin{aligned}
& \left(I_{0,+}^{\alpha, \alpha^{\prime}, \beta, \beta^{\prime}, \gamma} t^{\rho-1} \mathscr{W}_{p, b, c}(t)\right)(x) \\
& =\frac{x^{\rho+p-1}}{2^{p}} \frac{\Gamma(\rho+p) \Gamma\left(\rho+p+\gamma-\alpha-\alpha^{\prime}-\beta\right)}{\Gamma\left(\rho+p+\beta^{\prime}\right) \Gamma\left(\rho+p+\gamma-\alpha-\alpha^{\prime}\right) \Gamma(\kappa)} \frac{\Gamma\left(\rho+p+\beta^{\prime}-\alpha^{\prime}\right)}{\Gamma\left(\rho+p+\gamma-\alpha^{\prime}-\beta\right)} \\
& \quad \times{ }_{6} F_{7}\left[\begin{array}{c}
\frac{\rho+p}{2}, \frac{\rho+p+1}{2}, \frac{\rho+p+\gamma-\alpha-\alpha^{\prime}-\beta}{2}, \frac{\rho+p-\alpha-\alpha^{\prime}+\beta+1}{2}, \frac{\rho+p+\beta^{\prime}-\alpha^{\prime}}{2}, \frac{\rho+p+\beta^{\prime}-\alpha^{\prime}+1}{2} ; \\
\kappa, \frac{\rho+p+\beta^{\prime}}{2}, \frac{\rho+p+\beta^{\prime}+1}{2}, \frac{\rho+p+\gamma-\alpha-\alpha^{\prime}}{2}, \frac{\rho+p+\gamma-\alpha-\alpha^{\prime}+1}{2}, \\
\frac{\rho+p+\gamma-\alpha^{\prime}-\beta}{2}, \frac{\rho+p+\gamma-\alpha^{\prime}-\beta+1}{2}
\end{array}\right] .
\end{aligned}
$$

Proof. It is known that $\Gamma(z+k)=\Gamma(z)(z)_{k}$. Thus

$$
\begin{gathered}
\Gamma\left[\begin{array}{c}
\rho+p+2 k, \rho+p+\gamma-\alpha-\alpha^{\prime}-\beta+2 k, \rho+p+\beta^{\prime}-\alpha^{\prime}+2 k \\
\rho+p+\beta^{\prime}+2 k, \rho+p+\gamma-\alpha-\alpha^{\prime}+2 k, \rho+p+\gamma-\alpha^{\prime}-\beta+2 k, \kappa+k
\end{array}\right] \\
=\frac{\Gamma(\rho+p) \Gamma\left(\rho+p+\gamma-\alpha-\alpha^{\prime}-\beta\right) \Gamma\left(\rho+p+\beta^{\prime}-\alpha^{\prime}\right)}{\Gamma\left(\rho+p+\beta^{\prime}\right) \Gamma\left(\rho+p+\gamma-\alpha-\alpha^{\prime}\right) \Gamma\left(\rho+p+\gamma-\alpha^{\prime}-\beta\right) \Gamma(\kappa)} \\
\quad \times \frac{(\rho+p)_{2 k}\left(\rho+p+\gamma-\alpha-\alpha^{\prime}-\beta\right)_{2 k}\left(\rho+p+\beta^{\prime}-\alpha^{\prime}\right)_{2 k}}{\left(\rho+p+\beta^{\prime}\right)_{2 k}\left(\rho+p+\gamma-\alpha-\alpha^{\prime}\right)_{2 k}\left(\rho+p+\gamma-\alpha^{\prime}-\beta\right)_{2 k}(\kappa)_{k}} .
\end{gathered}
$$


Now apply (3.3) on the right-hand side of the above equation and then the result follows from (15). This completes the proof.

By adopting a similar method the next result can be obtained from (19); we omit the details.
Theorem 6. Let $\alpha, \alpha^{\prime}, \beta, \beta^{\prime}, \gamma, \rho, p, b, c \in \mathbb{C}$ such that $\kappa \neq 0,-1,-2, \ldots$. Suppose that $\operatorname{Re}(\gamma)>0$ and $\operatorname{Re}(\rho-p)<$ $1+\min \left\{\operatorname{Re}(-\beta), \operatorname{Re}\left(\alpha+\alpha^{\prime}-\gamma\right), \operatorname{Re}\left(\alpha+\beta^{\prime}-\gamma\right)\right\}$. Then

$$
\begin{aligned}
& \left(I_{0,+}^{\alpha, \alpha^{\prime}, \beta, \beta^{\prime}, \gamma} t^{\rho-1} \mathscr{W}_{p, b, c}\left(\frac{1}{t}\right)\right)(x) \\
& =\frac{x^{\rho-p-\alpha-\alpha^{\prime}+\gamma-1}}{2^{p}} \frac{\Gamma\left(\alpha+\alpha^{\prime}+p-\gamma-\rho+1\right) \Gamma\left(\alpha+\beta^{\prime}-\gamma+p-\rho+1\right) \Gamma(-\beta+p-\rho+1)}{\Gamma(p-\rho+1) \Gamma(\kappa) \Gamma\left(\alpha+\alpha^{\prime}+\beta^{\prime}+p-\gamma-\rho+1\right) \Gamma(\alpha-\beta+p-\rho+1)} \\
& \times_{6} F_{7}\left[\begin{array}{c}
\frac{\alpha+\alpha^{\prime}+p-\gamma-\rho+1}{2}, \frac{\alpha+\alpha^{\prime}+p+\gamma-\rho+2}{2}, \frac{\alpha+\beta^{\prime}+p-\gamma-\rho+1}{2}, \frac{\alpha+\beta^{\prime}+p-\gamma-\rho+2}{2}, \\
\kappa, \frac{p-\rho+1}{2}, \frac{p-\rho+2}{2}, \frac{\alpha+\alpha^{\prime}+\beta^{\prime}+p-\gamma-\rho+1}{2}, \frac{\alpha+\alpha^{\prime}+\beta^{\prime}+p-\gamma-\rho+2}{2}, \\
\frac{\alpha-\beta+p-\rho+1}{2}, \frac{\alpha-\beta+p-\rho+2}{2}
\end{array}\right] . \frac{c}{4 x^{2}}, .
\end{aligned}
$$

\section{Fractional Integration of Trigonometric Functions}

4.1. Cosine and Hyperbolic Cosine Functions. For all $b \in \mathbb{C}$, if $p=-b / 2$, then the generalized Bessel function $\mathscr{W}_{p, b, c}(z)$ has the form

$$
\begin{gathered}
\mathscr{W}_{-b / 2, b, c^{2}}(z)=\left(\frac{2}{z}\right)^{b / 2} \frac{\cos c z}{\sqrt{\pi}}, \\
\mathscr{W}_{-b / 2, b,-c^{2}}(z)=\left(\frac{2}{z}\right)^{b / 2} \frac{\cosh c z}{\sqrt{\pi}} .
\end{gathered}
$$

Hence the following results are a consequence of Theorems 3 and 4 , respectively.

Corollary 7. Let $\alpha, \alpha^{\prime}, \beta, \beta^{\prime}, \gamma, \rho, c \in \mathbb{C}$ such that $\operatorname{Re}(\gamma)>0$ and

$$
\operatorname{Re}(\rho)>\max \left\{0, \operatorname{Re}\left(\alpha-\alpha^{\prime}-\beta-\gamma\right), \operatorname{Re}\left(\alpha^{\prime}-\beta^{\prime}\right)\right\}
$$

Then

$$
\begin{aligned}
& \left(I_{0,+}^{\alpha, \alpha^{\prime}, \beta, \beta^{\prime}, \gamma} t^{\rho-1} \cos (c t)\right)(x) \\
& \left.=\pi^{1 / 2} x^{\rho-\alpha-\alpha^{\prime}+\gamma-1} \times{ }_{3} \psi_{4}\left[\begin{array}{r}
(\rho, 2),\left(\rho+\gamma-\alpha-\alpha^{\prime}-\beta, 2\right),\left(\rho+\beta^{\prime}-\alpha^{\prime}, 2\right) ; \\
\left(\rho+\beta^{\prime}, 2\right),\left(\rho+\gamma-\alpha-\alpha^{\prime}, 2\right),\left(\rho+\gamma-\alpha^{\prime}-\beta, 2\right),\left(\frac{1}{2}, 1\right)
\end{array}\right) \cdot \frac{c^{2} x^{2}}{4}\right], \\
& \left(I_{0,+}^{\alpha, \alpha^{\prime}, \beta, \beta^{\prime}, \gamma} t^{\rho-1} \cosh (c t)\right)(x) \\
& =\pi^{1 / 2} x^{\rho-\alpha-\alpha^{\prime}+\gamma-1} \times{ }_{3} \psi_{4}\left[\begin{array}{r}
(\rho, 2),\left(\rho+\gamma-\alpha-\alpha^{\prime}-\beta, 2\right),\left(\rho+\beta^{\prime}-\alpha^{\prime}, 2\right) ; \\
\left(\rho+\beta^{\prime}, 2\right),\left(\rho+\gamma-\alpha-\alpha^{\prime}, 2\right),\left(\rho+\gamma-\alpha^{\prime}-\beta, 2\right),\left(\frac{1}{2}, 1\right)
\end{array}\right)
\end{aligned}
$$


Proof. On setting $p=-b / 2$ and replacing $c$ by $c^{2}$ into (11) and using (37), we have

$$
\begin{aligned}
& \left(I_{0,+}^{\alpha, \alpha^{\prime}, \beta, \beta^{\prime}, \gamma} t^{\rho-1}\left(\frac{2}{t}\right)^{b / 2} \frac{\cos (c t)}{\sqrt{\pi}}\right)(x) \\
& \quad=\frac{x^{\rho-(b / 2)-\alpha-\alpha^{\prime}+\gamma-1}}{2^{-b / 2}} \times{ }_{3} \psi_{4}\left[\begin{array}{l}
\left(\rho-\frac{b}{2}, 2\right),\left(\rho-\frac{b}{2}+\gamma-\alpha-\alpha^{\prime}-\beta, 2\right)\left(\rho-\frac{b}{2}+\beta^{\prime}-\alpha^{\prime}, 2\right) ; \\
\left.\left(\rho-\frac{b}{2}+\beta^{\prime}, 2\right),\left(\rho-\frac{b}{2}+\gamma-\alpha-\alpha^{\prime}, 2\right),\left(\rho-\frac{b}{2}+\gamma-\alpha^{\prime}-\beta, 2\right),\left(\frac{1}{2}, 1\right) \mid-\frac{c x^{2}}{4}\right] .
\end{array}\right.
\end{aligned}
$$

This implies that

$$
\begin{aligned}
& \left(I_{0,+}^{\alpha, \alpha^{\prime}, \beta, \beta^{\prime}, \gamma} t^{\rho-(b / 2)-1} \cos c t\right)(x) \\
& \left.\quad=\pi^{1 / 2} x^{\rho-(b / 2)-\alpha-\alpha^{\prime}+\gamma-1} \times{ }_{3} \psi_{4}\left[\begin{array}{c}
\left(\rho-\frac{b}{2}, 2\right),\left(\rho-\frac{b}{2}+\gamma-\alpha-\alpha^{\prime}-\beta, 2\right)\left(\rho-\frac{b}{2}+\beta^{\prime}-\alpha^{\prime}, 2\right) ; \\
\left(\rho-\frac{b}{2}+\beta^{\prime}, 2\right),\left(\rho-\frac{b}{2}+\gamma-\alpha-\alpha^{\prime}, 2\right),\left(\rho-\frac{b}{2}+\gamma-\alpha^{\prime}-\beta, 2\right),\left(\frac{1}{2}, 1\right)
\end{array}\right] . \frac{c x^{2}}{4}\right] .
\end{aligned}
$$

The identity (39) follows from (30) by replacing $\rho$ by $\rho+(b / 2)$.

Similarly, the identity (4.7) can be obtained from (11) by setting $p=-b / 2$ and replacing $c$ by $-c^{2}$.

Corollary 8. Let $\alpha, \alpha^{\prime}, \beta, \beta^{\prime}, \gamma, \rho \in \mathbb{C}$ be such that $\operatorname{Re}(\gamma)>0$, and

$$
\operatorname{Re}(\rho)<\min \left\{\operatorname{Re}(-\beta), \operatorname{Re}\left(\alpha+\alpha^{\prime}-\gamma\right), \operatorname{Re}\left(\alpha+\beta^{\prime}-\gamma\right)\right\} .
$$

Then

$$
\begin{aligned}
& \left(I_{0,+}^{\alpha, \alpha^{\prime}, \beta, \beta^{\prime}, \gamma} t^{\rho-1} \cos \left(\frac{c}{t}\right)\right)(x) \\
& \left.=\pi^{1 / 2} x^{\rho-\alpha-\alpha^{\prime}+\gamma} \times{ }_{3} \psi_{4}\left[\begin{array}{c}
\left(\rho-\gamma+\alpha+\alpha^{\prime}, 2\right),\left(-\rho+\alpha+\beta^{\prime}-\gamma, 2\right),(-\rho-\beta, 2) ; \\
(-\rho, 2),\left(-\rho-\gamma+\alpha+\alpha^{\prime}+\beta^{\prime}, 2\right),(-\rho+\alpha-\beta, 2),\left(\frac{1}{2}, 1\right)
\end{array}\right) \frac{c^{2}}{4 x^{2}}\right], \\
& \left(I_{0,+}^{\alpha, \alpha^{\prime}, \beta, \beta^{\prime}, \gamma} t^{\rho} \cosh \left(\frac{c}{t}\right)\right)(x) \\
& \left.=\pi^{1 / 2} x^{\rho-\alpha-\alpha^{\prime}+\gamma} \times{ }_{3} \psi_{4}\left[\begin{array}{c}
\left(\rho-\gamma+\alpha+\alpha^{\prime}, 2\right),\left(-\rho+\alpha+\beta^{\prime}-\gamma, 2\right),(-\rho-\beta, 2) ; \\
(-\rho, 2),\left(-\rho-\gamma+\alpha+\alpha^{\prime}+\beta^{\prime}, 2\right),(-\rho+\alpha-\beta, 2),\left(\frac{1}{2}, 1\right)
\end{array}\right) \frac{c^{2}}{4 x^{2}}\right] .
\end{aligned}
$$

The next statements show that the image formulas for cosine and hyperbolic cosine under Saigo-Maeda fractional integral operators can also be represented in terms of the generalized hypergeometric series. This result follows from Theorems 5 and 6 with taking $p=-b / 2$ and replacing $c$ by $c^{2}$ or $-c^{2}$, respectively.
Corollary 9. Let $\alpha, \alpha^{\prime}, \beta, \beta^{\prime}, \gamma, \rho, c \in \mathbb{C}$. Suppose that $\operatorname{Re}(\gamma)>$ 0 and

$$
\operatorname{Re}(\rho)>\max \left\{0, \operatorname{Re}\left(\alpha-\alpha^{\prime}-\beta-\gamma\right), \operatorname{Re}\left(\alpha^{\prime}-\beta^{\prime}\right)\right\}
$$


Mathematical Problems in Engineering

7

Then the following formula holds:

$$
\begin{aligned}
& \left(I_{0,+}^{\alpha, \alpha^{\prime}, \beta, \beta^{\prime}, \gamma} t^{\rho-1} \cos (c t)\right)(x) \\
& =\frac{\Gamma(\rho) \Gamma\left(\rho+\gamma-\alpha-\alpha^{\prime}-\beta\right) \Gamma\left(\rho+\beta^{\prime}-\alpha^{\prime}\right)}{\Gamma\left(\rho+\beta^{\prime}\right) \Gamma\left(\rho+\gamma-\alpha-\alpha^{\prime}\right) \Gamma\left(\rho+\gamma-\alpha^{\prime}-\beta\right)} x^{\rho-\alpha-\alpha^{\prime}+\gamma-1} \\
& \times_{6} F_{7}\left[\begin{array}{c}
\frac{\rho}{2}, \frac{\rho+1}{2}, \frac{\rho+\gamma-\alpha-\alpha^{\prime}-\beta}{2}, \frac{\rho+\gamma-\alpha-\alpha^{\prime}+\beta+1}{2}, \frac{\rho+\beta^{\prime}-\alpha^{\prime}}{2}, \frac{\rho+\beta^{\prime}-\alpha^{\prime}+1}{2} ; \\
\frac{1}{2}, \frac{\rho+\beta^{\prime}}{2}, \frac{\rho+\beta^{\prime}+1}{2}, \frac{\rho+\gamma-\alpha-\alpha^{\prime}}{2}, \frac{\rho+\gamma-\alpha-\alpha^{\prime}+1}{2}, \frac{\rho+\gamma-\alpha^{\prime}-\beta}{2}, \frac{\rho+\gamma-\alpha^{\prime}-\beta+1}{2} \mid-\frac{c^{2} x^{2}}{4}
\end{array}\right], \\
& \left(I_{0,+}^{\alpha, \alpha^{\prime}, \beta, \beta^{\prime}, \gamma} t^{\rho-1} \cosh (c t)\right)(x) \\
& =\frac{\Gamma(\rho) \Gamma\left(\rho+\gamma-\alpha-\alpha^{\prime}-\beta\right) \Gamma\left(\rho+\beta^{\prime}-\alpha^{\prime}\right)}{\Gamma\left(\rho+\beta^{\prime}\right) \Gamma\left(\rho+\gamma-\alpha-\alpha^{\prime}\right) \Gamma\left(\rho+\gamma-\alpha^{\prime}-\beta\right)} x^{\rho-\alpha-\alpha^{\prime}+\gamma-1} \\
& \times_{6} F_{7}\left[\begin{array}{c}
\frac{\rho}{2}, \frac{\rho+1}{2}, \frac{\rho+\gamma-\alpha-\alpha^{\prime}-\beta}{2}, \frac{\rho+\gamma-\alpha-\alpha^{\prime}+\beta+1}{2}, \frac{\rho+\beta^{\prime}-\alpha^{\prime}}{2}, \frac{\rho+\beta^{\prime}-\alpha^{\prime}+1}{2} ; \\
\frac{1}{2}, \frac{\rho+\beta^{\prime}}{2}, \frac{\rho+\beta^{\prime}+1}{2}, \frac{\rho+\gamma-\alpha-\alpha^{\prime}}{2}, \frac{\rho+\gamma-\alpha-\alpha^{\prime}+1}{2}, \frac{\rho+\gamma-\alpha^{\prime}-\beta}{2}, \frac{\rho+\gamma-\alpha^{\prime}-\beta+1}{2} \mid \frac{c^{2} x^{2}}{4}
\end{array}\right] .
\end{aligned}
$$

Corollary 10. Let $\alpha, \alpha^{\prime}, \beta, \beta^{\prime}, \gamma, \rho, c \in \mathbb{C}$. Suppose that $\operatorname{Re}(\gamma)>0$ and

$$
\begin{aligned}
& \operatorname{Re}(\rho) \\
& \quad<1+\min \left\{\operatorname{Re}(-\beta), \operatorname{Re}\left(\alpha+\alpha^{\prime}-\gamma\right), \operatorname{Re}\left(\alpha+\beta^{\prime}-\gamma\right)\right\} .
\end{aligned}
$$

Then

$$
\begin{aligned}
& \left(I_{0,-}^{\alpha, \alpha^{\prime}, \beta, \beta^{\prime}, \gamma} t^{\rho} \cos \left(\frac{c}{t}\right)\right)(x) \\
& =\frac{\Gamma\left(\alpha+\alpha^{\prime}-\gamma-\rho\right) \Gamma\left(\alpha+\beta^{\prime}-\gamma-\rho\right) \Gamma(-\beta-\rho)}{\Gamma(-\rho) \Gamma\left(\alpha+\alpha^{\prime}+\beta^{\prime}-\gamma-\rho\right) \Gamma(\alpha-\beta-\rho)} x^{\rho-\alpha-\alpha^{\prime}+\gamma} \\
& \times_{6} F_{7}\left[\begin{array}{l}
\frac{\alpha+\alpha^{\prime}-\rho}{2}, \frac{\alpha+\alpha^{\prime}-\gamma-\rho+1}{2}, \frac{\alpha+\beta^{\prime}-\gamma-\rho}{2}, \frac{\alpha+\beta^{\prime}-\gamma-\rho+1}{2}, \frac{-\beta-\rho}{2}, \frac{-\beta-\rho+1}{2} ; \\
\frac{1}{2},-\frac{\rho}{2},-\frac{\rho+1}{2}, \frac{\alpha+\alpha^{\prime}+\beta^{\prime}-\gamma-\rho}{2}, \frac{\alpha+\alpha^{\prime}+\beta^{\prime}-\gamma-\rho+1}{2}, \frac{\alpha-\beta-\rho}{2}, \frac{\alpha-\beta-\rho+1}{2}, \mid-\frac{c^{2}}{4 x^{2}}
\end{array}\right] \text {, } \\
& \left(I_{0,-}^{\alpha, \alpha^{\prime}, \beta, \beta^{\prime}, \gamma} t^{\rho} \cosh \left(\frac{c}{t}\right)\right)(x) \\
& =\frac{\Gamma\left(\alpha+\alpha^{\prime}-\gamma-\rho\right) \Gamma\left(\alpha+\beta^{\prime}-\gamma-\rho\right)}{\Gamma(-\rho) \Gamma\left(\alpha+\alpha^{\prime}+\beta^{\prime}-\gamma-\rho\right)} \frac{\Gamma(-\beta-\rho)}{\Gamma(\alpha-\beta-\rho)} x^{\rho-\alpha-\alpha^{\prime}+\gamma} \\
& \times_{6} F_{7}\left[\begin{array}{c}
\frac{\alpha+\alpha^{\prime}-\rho}{2}, \frac{\alpha+\alpha^{\prime}-\gamma-\rho+1}{2}, \frac{\alpha+\beta^{\prime}-\gamma-\rho}{2}, \frac{\alpha+\beta^{\prime}-\gamma-\rho+1}{2}, \frac{-\beta-\rho}{2}, \frac{-\beta-\rho+1}{2} ; \\
\frac{1}{2},-\frac{\rho}{2},-\frac{\rho+1}{2}, \frac{\alpha+\alpha^{\prime}+\beta^{\prime}-\gamma-\rho}{2}, \frac{\alpha+\alpha^{\prime}+\beta^{\prime}-\gamma-\rho+1}{2}, \frac{\alpha-\beta-\rho}{2}, \frac{\alpha-\beta-\rho+1}{2} \mid \frac{c^{2}}{4 x^{2}}
\end{array}\right] .
\end{aligned}
$$


4.2. Sine and Hyperbolic Sine Functions. For all $b \in \mathbb{C}$, if $p=$ $1-b / 2$, then the generalized Bessel function $\mathscr{W}_{p, b, c}(z)$ has the form

$$
\begin{aligned}
\mathscr{W}_{1-(b / 2), b, c^{2}}(z) & =\left(\frac{2}{z}\right)^{b / 2} \frac{\sin (c z)}{\sqrt{\pi}}, \\
\mathscr{W}_{1-(b / 2), b,-c^{2}}(z) & =\left(\frac{2}{z}\right)^{b / 2} \frac{\sinh (c z)}{\sqrt{\pi}} .
\end{aligned}
$$

Thus, the composition of Saigo-Maeda fractional integral operators with sine and hyperbolic sine functions can be obtained from Theorems 3 and 4, respectively.
Corollary 11. Let $\alpha, \alpha^{\prime}, \beta, \beta^{\prime}, \gamma, \rho, c \in \mathbb{C}$ such that $\operatorname{Re}(\gamma)>0$ and

$$
\operatorname{Re}(\rho)>\max \left\{0, \operatorname{Re}\left(\alpha-\alpha^{\prime}-\beta-\gamma\right), \operatorname{Re}\left(\alpha^{\prime}-\beta^{\prime}\right)\right\} .
$$

$$
\begin{aligned}
& \left(I_{0,+}^{\alpha, \alpha^{\prime}, \beta, \beta^{\prime}, \gamma} t^{\rho-1} \sin (c t)\right)(x) \\
& \quad=\pi^{1 / 2} x^{\rho-\alpha-\alpha^{\prime}+\gamma-1} \times{ }_{3} \psi_{4}\left[\begin{array}{c}
(\rho, 2),\left(\rho+\gamma-\alpha-\alpha^{\prime}-\beta, 2\right),\left(\rho+\beta^{\prime}-\alpha^{\prime}, 2\right) ; \\
\left(\rho+\beta^{\prime}, 2\right),\left(\rho+\gamma-\alpha-\alpha^{\prime}, 2\right),\left(\rho+\gamma-\alpha^{\prime}-\beta, 2\right),\left(\frac{3}{2}, 1\right)
\end{array} \mid-\frac{c^{2} x^{2}}{4}\right], \\
& \left(I_{0,+}^{\alpha, \alpha^{\prime}, \beta, \beta^{\prime}, \gamma} t^{\rho-1} \sinh (c t)\right)(x) \\
& =\pi^{1 / 2} x^{\rho-\alpha-\alpha^{\prime}+\gamma-1} \times{ }_{3} \psi_{4}\left[\begin{array}{c}
(\rho, 2),\left(\rho+\gamma-\alpha-\alpha^{\prime}-\beta, 2\right),\left(\rho+\beta^{\prime}-\alpha^{\prime}, 2\right) ; \\
\left(\rho+\beta^{\prime}, 2\right),\left(\rho+\gamma-\alpha-\alpha^{\prime}, 2\right),\left(\rho+\gamma-\alpha^{\prime}-\beta, 2\right),\left(\frac{3}{2}, 1\right)
\end{array} \mid \frac{c^{2} x^{2}}{4}\right] .
\end{aligned}
$$

The next result follows from Theorem 4 by setting $p=$ $-b / 2$ and replacing $c$ by $c^{2}$ or $-c^{2}$, respectively.
Corollary 12. Let $\alpha, \alpha^{\prime}, \beta, \beta^{\prime}, \gamma, \rho \in \mathbb{C}$ be such that $\operatorname{Re}(\gamma)>0$ and

$$
\operatorname{Re}(\rho)<\min \left\{\operatorname{Re}(-\beta), \operatorname{Re}\left(\alpha+\alpha^{\prime}-\gamma\right), \operatorname{Re}\left(\alpha+\beta^{\prime}-\gamma\right)\right\} .
$$

Then

$$
\begin{aligned}
& \left(I_{0,+}^{\alpha, \alpha^{\prime}, \beta, \beta^{\prime}, \gamma^{\rho-1}} \sin \left(\frac{c}{t}\right)\right)(x) \\
& \left.=\pi^{1 / 2} x^{\rho-\alpha-\alpha^{\prime}+\gamma} \times{ }_{3} \psi_{4}\left[\begin{array}{c}
\left(\rho-\gamma+\alpha+\alpha^{\prime}, 2\right),\left(-\rho+\alpha+\beta^{\prime}-\gamma, 2\right),(-\rho-\beta, 2) ; \\
(-\rho, 2),\left(-\rho-\gamma+\alpha+\alpha^{\prime}+\beta^{\prime}, 2\right),(-\rho+\alpha-\beta, 2),\left(\frac{3}{2}, 1\right)
\end{array}\right) \mid-\frac{c^{2}}{4 x^{2}}\right], \\
& \left(I_{0,+}^{\alpha, \alpha^{\prime}, \beta, \beta^{\prime}, \gamma^{\rho}} t^{\rho} \sinh \left(\frac{c}{t}\right)\right)(x) \\
& \left.=\pi^{1 / 2} x^{\rho-\alpha-\alpha^{\prime}+\gamma} \times{ }_{3} \psi_{4}\left[\begin{array}{c}
\left(\rho-\gamma+\alpha+\alpha^{\prime}, 2\right),\left(-\rho+\alpha+\beta^{\prime}-\gamma, 2\right),(-\rho-\beta, 2) ; \\
(-\rho, 2),\left(-\rho-\gamma+\alpha+\alpha^{\prime}+\beta^{\prime}, 2\right),(-\rho+\alpha-\beta, 2),\left(\frac{3}{2}, 1\right)
\end{array}\right) \frac{c^{2}}{4 x^{2}}\right]
\end{aligned}
$$

The following result can be obtained from Theorems 5 and 6 with taking $p=-b / 2$ and replacing $c$ by $c^{2}$ or $-c^{2}$, respectively.
Corollary 13. Let $\alpha, \alpha^{\prime}, \beta, \beta^{\prime}, \gamma, \rho, c \in \mathbb{C}$. Suppose that $\operatorname{Re}(\gamma)>0$ and

$$
\operatorname{Re}(\rho)>\max \left\{0, \operatorname{Re}\left(\alpha-\alpha^{\prime}-\beta-\gamma\right), \operatorname{Re}\left(\alpha^{\prime}-\beta^{\prime}\right)\right\} .
$$


Then the following formula holds:

$$
\left.\begin{array}{l}
\left(I_{0,+}^{\alpha, \alpha^{\prime}, \beta, \beta^{\prime}, \gamma} t^{\rho-1} \sin (c t)\right)(x) \\
=\frac{\Gamma(\rho) \Gamma\left(\rho+\gamma-\alpha-\alpha^{\prime}-\beta\right) \Gamma\left(\rho+\beta^{\prime}-\alpha^{\prime}\right)}{\Gamma\left(\rho+\beta^{\prime}\right) \Gamma\left(\rho+\gamma-\alpha-\alpha^{\prime}\right) \Gamma\left(\rho+\gamma-\alpha^{\prime}-\beta\right)} x^{\rho-\alpha-\alpha^{\prime}+\gamma-1} \\
\quad \times{ }_{6} F_{7}\left[\frac{3}{2}, \frac{\rho+\beta^{\prime}}{2}, \frac{\rho+\beta^{\prime}+1}{2}, \frac{\rho+\gamma-\alpha-\alpha^{\prime}}{2}, \frac{\rho+\gamma-\alpha-\alpha^{\prime}+1}{2}, \frac{\rho+\gamma-\alpha^{\prime}-\beta}{2}, \frac{\rho+\gamma-\alpha^{\prime}-\beta+1}{2} ;\right.
\end{array}\right]
$$

Corollary 14. Let $\alpha, \alpha^{\prime}, \beta, \beta^{\prime}, \gamma, \rho, c \in \mathbb{C}$. Suppose that $\operatorname{Re}(\gamma)>0$ and $\operatorname{Re}(\rho)<1+\min \left\{\operatorname{Re}(-\beta), \operatorname{Re}\left(\alpha+\alpha^{\prime}-\gamma\right), \operatorname{Re}(\alpha+\right.$ $\left.\left.\beta^{\prime}-\gamma\right)\right\}$. Then

$$
\begin{aligned}
& \left(I_{0,-}^{\alpha, \alpha^{\prime}, \beta, \beta^{\prime}, \gamma} t^{\rho} \sin \left(\frac{c}{t}\right)\right)(x) \\
& =\frac{\Gamma\left(\alpha+\alpha^{\prime}-\gamma-\rho\right) \Gamma\left(\alpha+\beta^{\prime}-\gamma-\rho\right)}{\Gamma(-\rho) \Gamma\left(\alpha+\alpha^{\prime}+\beta^{\prime}-\gamma-\rho\right)} \frac{\Gamma(-\beta-\rho)}{\Gamma(\alpha-\beta-\rho)} x^{\rho-\alpha-\alpha^{\prime}+\gamma} \\
& \times_{6} F_{7}\left[\begin{array}{l}
\frac{\alpha+\alpha^{\prime}-\rho}{2}, \frac{\alpha+\alpha^{\prime}-\gamma-\rho+1}{2}, \frac{\alpha+\beta^{\prime}-\gamma-\rho}{2}, \frac{\alpha+\beta^{\prime}-\gamma-\rho+1}{2}, \frac{-\beta-\rho}{2}, \frac{-\beta-\rho+1}{2} ; \\
\frac{3}{2},-\frac{\rho}{2},-\frac{\rho+1}{2}, \frac{\alpha+\alpha^{\prime}+\beta^{\prime}-\gamma-\rho}{2}, \frac{\alpha+\alpha^{\prime}+\beta^{\prime}-\gamma-\rho+1}{2}, \frac{\alpha-\beta-\rho}{2}, \frac{\alpha-\beta-\rho+1}{2}, \mid-\frac{c^{2}}{4 x^{2}}
\end{array}\right], \\
& \left(I_{0,-}^{\alpha, \alpha^{\prime}, \beta, \beta^{\prime}, \gamma} t^{\rho} \sinh \left(\frac{c}{t}\right)\right)(x) \\
& =\frac{\Gamma\left(\alpha+\alpha^{\prime}-\gamma-\rho\right) \Gamma\left(\alpha+\beta^{\prime}-\gamma-\rho\right)}{\Gamma(-\rho) \Gamma\left(\alpha+\alpha^{\prime}+\beta^{\prime}-\gamma-\rho\right)} \frac{\Gamma(-\beta-\rho)}{\Gamma(\alpha-\beta-\rho)} x^{\rho-\alpha-\alpha^{\prime}+\gamma} \\
& \times_{6} F_{7}\left[\begin{array}{l}
\frac{\alpha+\alpha^{\prime}-\rho}{2}, \frac{\alpha+\alpha^{\prime}-\gamma-\rho+1}{2}, \frac{\alpha+\beta^{\prime}-\gamma-\rho}{2}, \frac{\alpha+\beta^{\prime}-\gamma-\rho+1}{2}, \frac{-\beta-\rho}{2}, \frac{-\beta-\rho+1}{2} ; \\
\frac{3}{2},-\frac{\rho}{2},-\frac{\rho+1}{2}, \frac{\alpha+\alpha^{\prime}+\beta^{\prime}-\gamma-\rho}{2}, \frac{\alpha+\alpha^{\prime}+\beta^{\prime}-\gamma-\rho+1}{2}, \frac{\alpha-\beta-\rho}{2}, \frac{\alpha-\beta-\rho+1}{2} \mid \frac{c^{2}}{4 x^{2}}
\end{array}\right] .
\end{aligned}
$$




\section{Concluding Observations}

In this section some consequences of the main result derived in previous sections are given in detail. Also comparison with other known results from the literature is listed.

(1) We remark that all the results given by Purohit et al. [10] are followed from the results derived in this paper by setting $b=1=c$.

(2) The results in Sections 2 and 3 also provide the Marichev-Saigo-Maeda fractional integration of modified Bessel function and spherical Bessel functions.

(3) Set $\alpha^{\prime}=0$ in the operators (1) and (2). Then due to identities given by Saxena and Saigo [11, P. 93], it follows that

$$
\begin{aligned}
& \left(I_{0,+}^{\alpha, 0, \beta, \beta^{\prime}, \gamma} f\right)(x)=\left(I_{0, x}^{\gamma, \alpha-\gamma,-\beta} f\right)(x) \\
& \left(I_{0,-}^{\alpha, 0, \beta, \beta^{\prime}, \gamma} f\right)(x)=\left(I_{x, \infty}^{\gamma, \alpha-\gamma,-\beta} f\right)(x) .
\end{aligned}
$$

The generalized integral transforms that appear in the right-hand side of the above equations are due to Saigo [19] and defined as follows:

$$
\begin{aligned}
&\left(I_{0_{+}}^{\alpha, \beta, \eta} f\right)(x)=\frac{x^{-\alpha-\beta}}{\Gamma(\alpha)} \int_{0}^{x}(x-t)^{\alpha-1}{ }_{2} F_{1} \\
& \times\left(\alpha+\beta,-\eta ; \alpha ; 1-\frac{t}{x}\right) f(t) d t \\
&\left(I_{-}^{\alpha, \beta, \eta} f\right)(x)=\frac{1}{\Gamma(\alpha)} \int_{x}^{\infty}(t-x)^{\alpha-1} t^{-\alpha-\beta}{ }_{2} F_{1} \\
& \quad \times\left(\alpha+\beta,-\eta ; \alpha ; 1-\frac{x}{t}\right) f(t) d t
\end{aligned}
$$

where $\Gamma(\alpha)$ is the Euler gamma function [20] and ${ }_{2} F_{1}(a, b ; c ; x)$ is the Gauss hypergeometric function.

The above fact helps us to conclude that all the results given in $[9,21]$ can also be obtained from the results in this paper by setting $\alpha^{\prime}=0$.

(4) Note that Riemann-Liouville and Weyl and ErdélyiKober fractional calculus [22] are special case of Saigo's operator (46). Thus this paper is also useful to derive certain composition formula involving Riemann-Liouville and Weyl and ErdléyiKober fractional calculus and Bessel, modified Bessel, and spherical Bessel function of the first kind.

\section{Conflict of Interests}

The authors declare that there is no conflict of interests regarding the publication of this paper.

\section{Acknowledgments}

The research work presented here was supported in part by the Deanship of Scientific Research (DSR) at Salman bin Abdulaziz University for K. S. Nisar.

\section{References}

[1] O. I. Marichev, "Volterra equation of Mellin convolution type with a Horn function in the kernel," Izvestiya Akademii Nauk BSSR. Seriya Fiziko-Matematicheskikh Nauk, vol. 1, pp. 128-129, 1974 (Russian).

[2] M. Saigo and N. Maeda, "More generalization of fractional calculus," in Transform Methods \& Special Functions, Varna '96, pp. 386-400, Bulgarian Academy of Sciences, Bulgaria, Sofia, 1998.

[3] Y. C. Kim, K. S. Lee, and H. M. Srivastava, "Some applications of fractional integral operators and Ruscheweyh derivatives," Journal of Mathematical Analysis and Applications, vol. 197, no. 2, pp. 505-517, 1996.

[4] V. Kiryakova, "All the special functions are fractional differintegrals of elementary functions," Journal of Physics A: Mathematical and General, vol. 30, no. 14, pp. 5085-5103, 1997.

[5] K. S. Miller and B. Ross, An Introduction to the Fractional Calculus and Fractional Differential Equations, John Wiley \& Sons, New York, NY, USA, 1993.

[6] H. M. Srivastava, S.-D. Lin, and P.-Y. Wang, "Some fractionalcalculus results for the $\bar{H}$-function associated with a class of Feynman integrals," Russian Journal of Mathematical Physics, vol. 13, no. 1, pp. 94-100, 2006.

[7] Á. Baricz, "Geometric properties of generalized Bessel functions," Publicationes Mathematicae, vol. 73, no. 1-2, pp. 155-178, 2008.

[8] S. R. Mondal and A. Swaminathan, "Geometric properties of generalized Bessel functions," Bulletin of the Malaysian Mathematical Sciences Society, vol. 35, no. 1, pp. 179-194, 2012.

[9] A. A. Kilbas and N. Sebastian, "Generalized fractional integration of Bessel function of the first kind," Integral Transforms and Special Functions, vol. 19, no. 11-12, pp. 869-883, 2008.

[10] S. D. Purohit, D. L. Suthar, and S. L. Kalla, "Marichev-SaigoMaeda fractional integration operators of the Bessel functions," Le Matematiche, vol. 67, no. 1, pp. 21-32, 2012.

[11] R. K. Saxena and M. Saigo, "Generalized fractional calculus of the $H$-function associated with the Appell function $F_{3}$," Journal of Fractional Calculus, vol. 19, pp. 89-104, 2001.

[12] C. Fox, "The asymptotic expansion of generalized hypergeometric functions," Proceedings of the London Mathematical Society, vol. s2-27, no. 1, pp. 389-400.

[13] E. M. Wright, "The asymptotic expansion of integral functions defined by Taylor series," Philosophical Transactions of the Royal Society of London A: Mathematical and Physical Sciences, vol. 238, pp. 423-451, 1940.

[14] E. M. Wright, "The asymptotic expansion of the generalized hypergeometric function," Proceedings of the London Mathematical Society, vol. 46, pp. 389-408, 1940.

[15] A. A. Kilbas, M. Saigo, and J. J. Trujillo, "On the generalized Wright function," Fractional Calculus \& Applied Analysis, vol. 5, no. 4, pp. 437-460, 2002.

[16] A. A. Kilbas and N. Sebastian, "Fractional integration of the product of Bessel functions of the first kind," Fractional Calculus \& Applied Analysis, vol. 13, no. 2, pp. 159-175, 2010.

[17] H. M. Srivastava, "Some Fox-Wright generalized hypergeometric functions and associated families of convolution operators," Applicable Analysis and Discrete Mathematics, vol. 1, no. 1, pp. 56-71, 2007.

[18] A. Erdélyi, W. Magnus, F. Oberhettinger, and F. G. Tricomi, Higher Transcendental Functions, vol. I, II, McGraw-Hill Book Company, New York, NY, USA, 1953. 
[19] M. Saigo, "A remark on integral operators involving the Gauss hypergeometric functions," Mathematical Reports of College of General Education, vol. 11, no. 2, pp. 135-143, 1978.

[20] E. D. Rainville, Special Functions, The Macmillan, New York, NY, USA, 1960.

[21] P. Malik, S. R. Mondal, and A. Swaminathan, "Fractional integration of generalized Bessel function of the first kind," in Proceedings of the ASME International Design Engineering Technical Conferences \& Computers and Information in Engineering Conference (IDETC/CIE '11), Washington, Wash, USA, August 2011.

[22] S. G. Samko, A. A. Kilbas, and O. I. Marichev, Fractional Integrals and Derivatives, Gordon and Breach Science Publishers, Yverdon, 1993, Translated from the 1987 Russian original. 


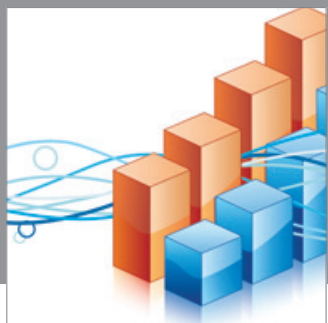

Advances in

Operations Research

mansans

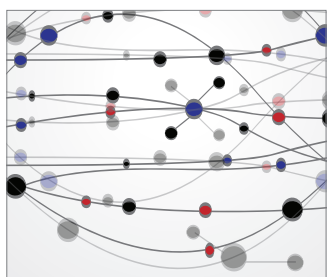

The Scientific World Journal
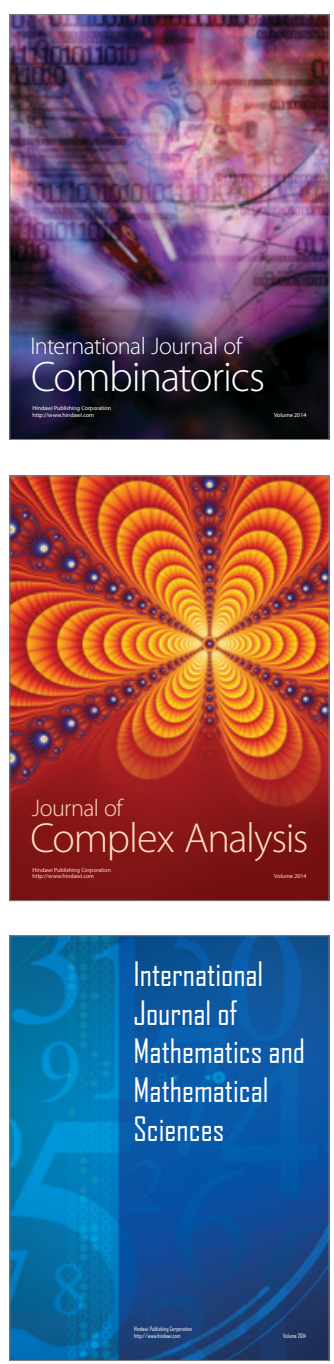
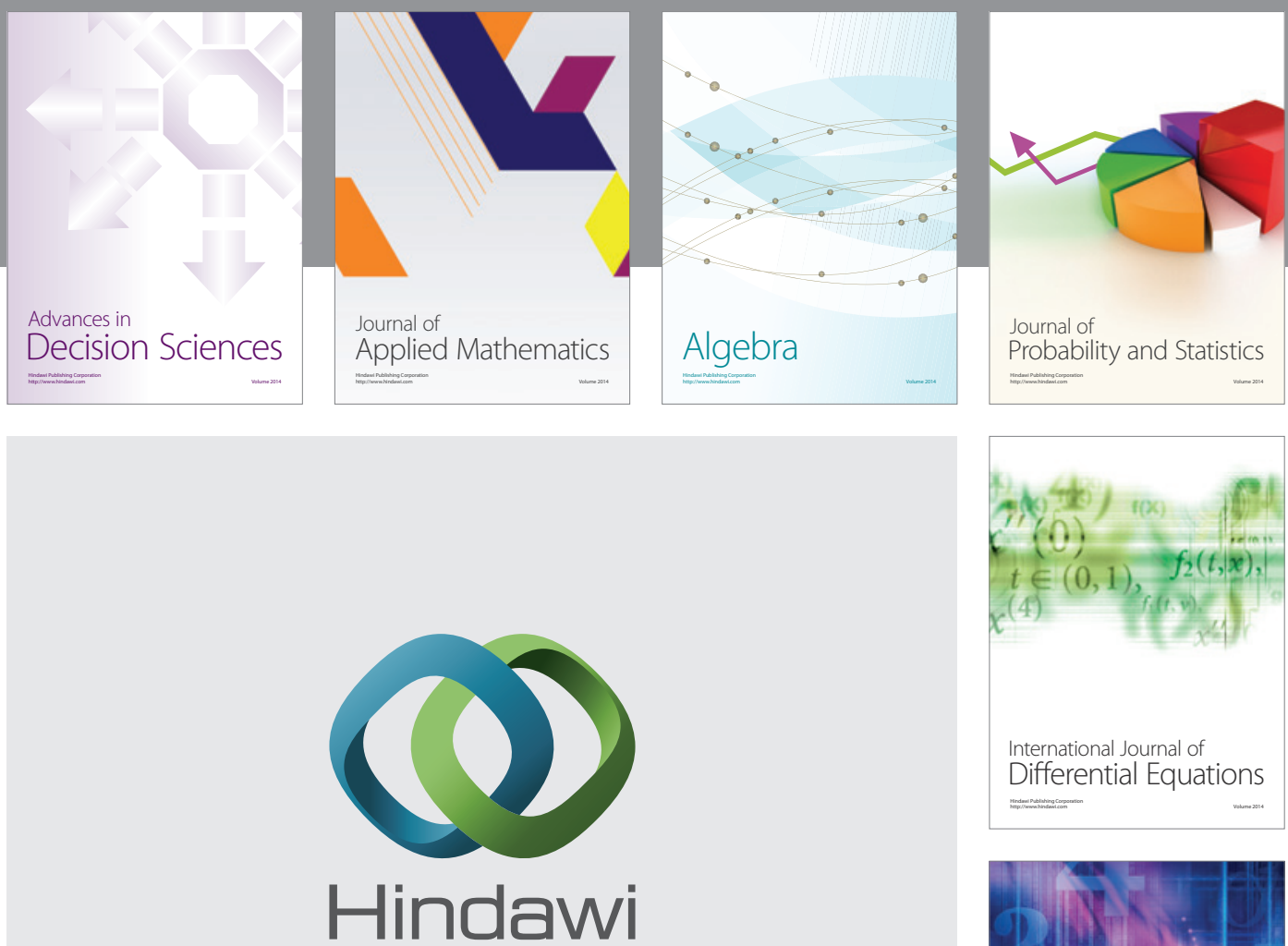

Submit your manuscripts at http://www.hindawi.com
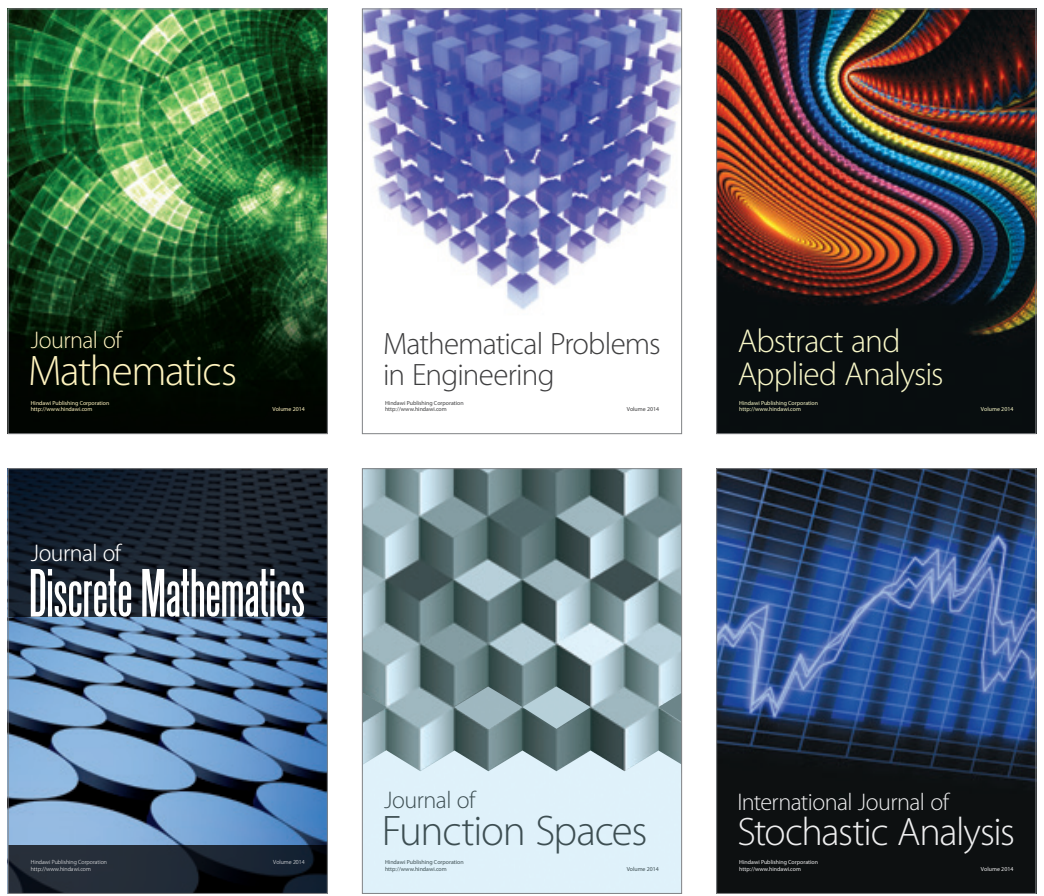

Journal of

Function Spaces

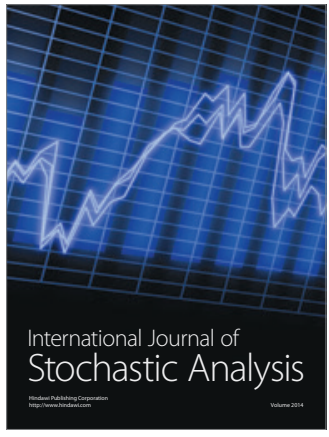

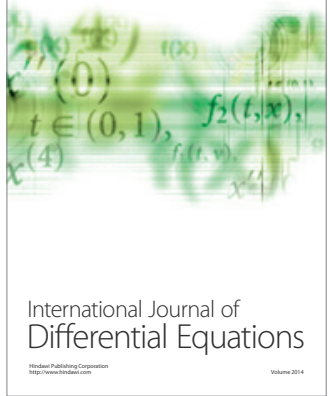
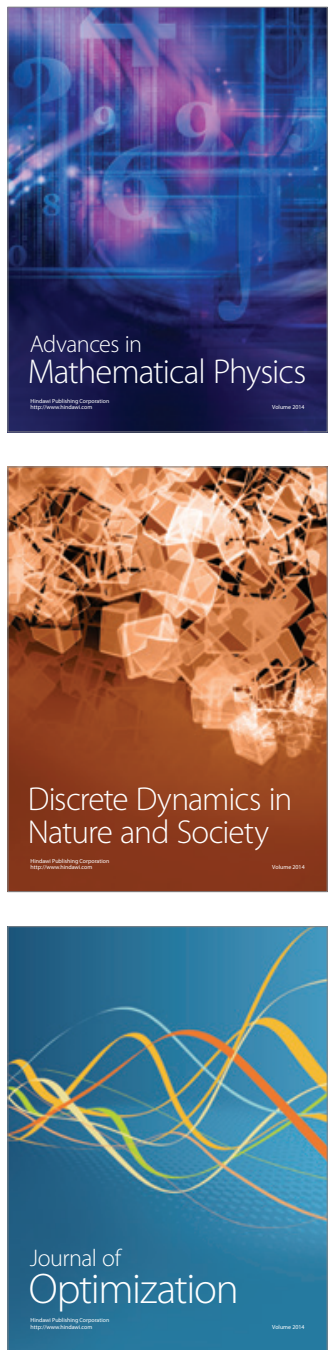